\title{
Study of Contemporary college student's psychological development characteristics and the patterns of sexual health education
}

\author{
Ru Lixian \\ school Hospital, Qilu Industrial University, Jinan , 250100, China. \\ email:rulixian1978@163.com
}

Keywords: College student; Sexual psychology; Sexual health education model

\begin{abstract}
Purpose: To understand contemporary college students psychological development present situation, to explore the sexual health of the existence of sexual psychological development features and hidden dangers, to guide college students' sexual health education pattern, to promote the development of college students mental health. Methods: 600 college students were randomly selected as the object of investigation, filled in the questionnaire of psychological health survey, including sexual cognition, adaptation, and heterosexual contacts view, sexual dream, sexual fantasy, sexual impulse and attitudes towards premarital sex. Finally, we counted results by using SPSS13.0 statistical software. Results: Scores of "itself in the development which changes" was highest, but sexually transmitted diseases and contraceptive knowledge understanding degree were low; "I can active and effective use of the society, families, schools, provide information and access to sexual knowledge" had the highest scores, "I can pass the proper way to eliminate sexual desire, arousal," scored lowest; the attitude to opposite sex association held by the men and women had certain differences, said 52\% of the boys had a strong desire to heterosexual contacts, while girl was $33.3 \%, 4.3 \%$ of all the boys were opposition to heterosexual contacts , while the girl was $12.28 \%$; "sometimes" sexual dream, sexual fantasy, sexual impulse were about $40 \%$, the proportion of the often less than $20 \%$, about $15 \%$ of college students said never appeared above phenomenon; Attitude towards premarital sex survey, the results showed that the boys voted, by significant difference comparing with girls had. Conclusion: On the whole , contemporary university students' sexual psychology was healthier, but comprehensive sexual cognition was not enough, sexual attitude was open, and there were some hidden dangers. So students' sexual health education is imminent, formulating a set of education model suitable for college student's psychological development features is necessary.
\end{abstract}

\section{Introduction}

College student is the group with love and sexual more formation and rapid development, and is also the group with the sexual physiology and psychology maturing [1]. Opinions vary about the definition of sexual psychology. SONG Shuwen, YUAN Zhi qi consider " Sexual psychology is one of the branch of psychology, with the research object as people in the sex psychological activity rules, main content including physiological and psychological foundation of male and female behavior, differences in the development of the sexual consciousness of all ages, sound and exception sexual psychology, sexual disorders of psychological causes and psychological treatment, the sexual consciousness in family planning, etc.Sexual psychology has the closest relations with physiology, sex psychology and youth psychology " [2]. ZHANG Jinfu think "sexual psychology is a problem about sex psychological activity, is reflect the main body to sexual physiology, sexual object and the relations between the sexes, also calls the sex consciousness. Sexual psychology involves all mental activities of sexual knowledge, sexual emotion and sexual experience, sexual behavior "[4]. About the meaning of sexual health, (WHO) publication of the research progress of reproductive health put forward: sexual health is a kind of sex associating with physical, emotional, mental and social well-being: it not only means to get rid of illness, disability or weak [5]. College student is in the rapid development period of physiological and psychological, but sexual 
psychological maturity lags behind the development of sexual physiology. The problem of "sex" is still difficult to say in higher education combined with the influence of social morals, the culture and the surrounding environment. In society, it is difficult to see a sex education books that are suitable for college students, in the family, Rarely talked about, or failing to lead. All the above facts lead to lack of health knowledge of college students generally [6]. Some scholars found that college students lacked the knowledge of physiological knowledge and sexual health at large [8]. The contradiction between growing demand for health knowledge sex and reproductive health knowledge and information, education and service, and supportive environment serious lack of supply, brought college students a variety of sexual and reproductive health problems, which mainly includes an unwanted pregnancy, abortion, sexually transmitted diseases, sexual violence and so on $[9,10]$. The WHO survey think that the cause of the phenomenon, such as unmarried pregnancy and no protective behavior ,is the sex blind [11]. The sexual health education can effectively to solve these problems[13]. This study aims to adjust college students' sexual health education teaching mode on the basis of investigation and analysis, in order to adapt to the university students' sexual psychology and the characteristics of the sexual health development.

\section{Materials and methods}

600 respondents were selected from Qilu industrial university undergraduate students randomly ,with the age of 18 to 23 years old. A total of 600 questionnaires were provided, recycling585 effective questionnaire, including 300 boys and 285 girls, the recovery rate was 97.5\%.

Questionnaire consulted the related content of college students psychological research of the Chinese contemporary culture and "sexual psychology",as well as related content of "college students' sexual health questionnaire" written by sexual health education research association in Beijing, selected the high frequency of the same kind of research topic in recent years constitute a questionnaire. Questionnaire content mainly included five aspects, sexual cognition, adaptation with the opposite sex, sexual dream, sexual fantasy, sexual impulse and perceptions of premarital sex, Questionnaire was divided into two types. One is rating scale type title, divided into five levels, from low to high score of $1,2,3,4,5$ points respectively. The other is a can of multiple-choice questions. Investigators distributed questionnaires, introduced the attentive matters, provided the investigators' right to know, and took security measures to subjects bearer. Questionnaires were enclosed to the envelope respectively and recycled at the scene. A database of all the information was built by EXCEL and analyzed using SPSS 13.0 statistical.

\section{Results}

\section{Sexual cognition}

In this item, there was no significant difference among gender ,"I know body's change will appear in the process of development which "had the highest score, averaging 4.2 points, but "know the genital diseases" scored lowest, average score of 3.41, detailed statistical results are shown in table 1 . 
Table 1 Sexual knowledge item scores

survey items

$\begin{array}{rr}\text { average } & \begin{array}{c}\text { Standard } \\ \text { deviation }\end{array} \\ 4.01 & 0.79 \\ 3.55 & 1.12 \\ 3.89 & 0.98 \\ 4.2 & 0.71 \\ & \\ 3.9 & 0.99 \\ 3.62 & 1.09 \\ 3.41 & 1.23 \\ 3.58 & 1.11 \\ 3.99 & 0.95\end{array}$

To understand menstruation and spermatorrhea

To understand sexual dream, sexual fantasy, sexual impulse

To understand masturbation

To understand body will appear which changes in the

process of development

To understand the structure and functions of the

reproductive organs

To understand the knowledge about sexually transmitted diseases

To understand genital diseases

To understand the various contraceptive knowledge

To understand what is sexual harassment and hurt

\section{Sexual adaptation}

Sexual adaptation is that teenagers can happily accept their sexual characteristics change, good for gender identity, and constraint and adjust their sexual desire and sexual activity consciously, according to the requirement of the social and cultural .In 6 survey items, "I can use actively and effectively the resources of the society, the family, the school providing to access sexual knowledge" was the highest score, "I can pass the proper way to eliminate sexual desire, arousal," scored lowest. Concrete results are shown in table 2.

Table 2 Sexual adaptation item scores

survey items average standard deviation

to eliminate sexual desire, sexual impulse through the proper way

3.52

the sexual behavior to be accord with the requirement of the environment

the approach resulting sexual gratification is conforming to the moral norms of the society

sexual behavior conform to social cultural background at that time

access to sexual knowledge from the resources of society, family,

school actively and effectively

4.01

can try my best to transfer sexual impulse and sexual desire

3.88

\section{Wiew with the opposite sex}

In this survey, the attitude held by the men and women had certain differences, $52 \%$ of the boys had a strong desire for heterosexual contacts, while girls (33.3\%).4.3\% of boys didn't want to associate with the opposite sex, while the girls were $12.28 \%$. the results are shown in table 3 .

Table 3 willing to contact with opposite sex(percentage)

survey items

Very willing to

willing to

It doesn't matter

Don't want to

$\begin{array}{llr}\text { man } & \text { woman } & \text { headcount } \\ 156(52) & 95(33.3) & 251 \\ 101(33.67) & 102(35.79) & 203 \\ 30(10) & 53(18.6) & 83 \\ 13(4.3) & 35(12.28) & 48\end{array}$

\section{Sexual fantasy, sexual dream, sexual impulse}

Sexual fantasy is refers to imagination that the people are awake to cannot achieve the sexual matters, is a "serial story" with sexual color, is mankind's common phenomenon. Sexual dreams is to point to dreams with the opposite sex, is also a kind of common normal psychological phenomenon. Sexual arousal is refers to the sex desire and impulse under the joint action of the sex hormones and the environmental stimuli both inside and outside. the result can appear sometimes sexual dream, sexual fantasy, sexual arousal is around $40 \%$, the proportion of the often less than $20 \%$, about $15 \%$ of college students said never appear above phenomenon. The specific results are shown in table 4. 
Table 4 investigation on Sexual fantasy, sexual dream, sexual impulse (percentage)

$\begin{array}{llll}\begin{array}{l}\text { survey items } \\ \text { often }\end{array} & \text { Sexual fantasy } & \text { sexual dream } & \text { sexual impulse } \\ \text { Sometimes } & 98(16.75) & 121(20.68) & 101(17.26) \\ \text { now and then } & 225(38.46) & 235(40.17) & 256(46.13) \\ \text { nothing } & 178(30.43) & 165(28.21) & 132(22.56) \\ \end{array}$

\section{Attitude to premarital sex}

This survey found that attitude to premarital sex of boys and girls had significant difference. Boy's attitude to premarital sex were more relaxed, which rate of first four approval was close to $60 \%$, while girl's was $22 \%$. $16.3 \%$ of boys had disapprove of premarital sex, while women as much as $65.6 \%$ had ,as they thought it is harm for the parties and immoral, concrete results are shown in table 5 .

Table 5 investigation on attitude to premarital sex survey(percentage)

survey items

As long as both love each other ,It can

As long as two people willing to ,it can

as long as they are not pregnant, it can

As long as they get married ,it can

it is physiological and psychological demand of the

college students

Will have harm to the parties, can not

Is unethical behavior, can not

$\begin{array}{lll}\text { man } & \text { woman } & \text { headcount } \\ 42(14) & 27(9.47) & 69(11.79) \\ 35(11.67) & 10(3.51) & 45(7.69) \\ 40(13.33) & 10(3.51) & 50(8.55) \\ 56(18.67) & 15(5.26) & 71(12.14) \\ 78(26) & 36(12.63) & 114(19.49) \\ 35(11.67) & 101(35.44) & 136(23.25) \\ 14(4.67) & 86(30.18) & 100(17.09)\end{array}$

\section{Discussion}

4.1 Generally speaking, sex psychological development accords with the general law of this age. College student is generally between 17 to 23 years old, sexual physiology and psychology have been mature gradually. The sex disgust had passed and entered the stage of sexual attraction. They are eager to show the perfect myself in front of the opposite sex, and desire for the attention of the opposite sex. Most of the students in this study are willing to associate with the opposite sex. It conforms to the characteristics of psychological development.

4.2 Sexual knowledge is not enough comprehensive, the channel acquiring knowledge is not normal. According to the survey, the phenomenon such as unmarried pregnancy, abortion and sexually transmitted diseases is on the rise among college students in recent years [15], repeated abortion will badly hurt to the female body ,not only easy to reproductive system disease, but severe cases can lead to secondary infertility, it can adverse influence on normal family life after marriage. Meanwhile some unorthodox channels of knowledge acquisition are given priority to, such as network, pornographic books, and film and television works. Some researchers have found that the rate that college students obtain scientific knowledge from the school formal way is still rarely [16-18], the main source for the network, a movie, with learning, school education account for only $10 \%$ to $30 \%(19,20)$. As college students lack the ability of the authenticity with things complicated on the network, it will be directed some wrong information, even to the crime.

4.3 Sexual consciousness is awakening, and more open concept in sex. Modern college students are the sexual consciousness awakening early and more open concept in sex under the influence of western "sex". From above survey results on sexual fantasies and impulsive, and premarital sex, it can be concluded that college students' attitude towards premarital sex is loose, especially boys, think that as long as there is a feeling, two people are willing to, or is married for the purpose of sex, it is acceptable and agreed. Sexual impulse and sexual fantasies are more common, but the way to send sexual impulse also is not very clear. So college students are confused. It is the main confusion in the stage of university students, because they don't know how to 
correctly treat sexual arousal, sometime they can take some mistakes, even extreme way, sometimes also can appear sex crimes.

\section{Prospects}

Psychological development of college student has its uniqueness. It is necessary to correctly guide, so is necessary to strengthen college students' sexual health education. The sexual health education is at the initial stage, is mostly quoted foreign sexual health education theory and model. At present, there are three main modes in the Us and Europe, abstinence-only sex education mode, comprehensive education, sex education in Europe. Domestic scholars is biased toward comprehensive education and sex education in Europe [22], emphasize to understand sex under physical, psychological social, in different cultural, racial, and religious background ,to set up the attitudes towards sex, to advocate personal beliefs choose autonomy and the absolute moralization. In Europe, the concept of sexual health education more widely included as the content of as much as possible about sex [23], and sex education is the process throughout the lifetime of concept development, cultivation of attitudes, beliefs, and values to confirm [24]. Domestic adolescent sexual health education has just started, also did not form an unified teaching modes, now is still in the exploratory stage. Some scholars constructed the course - activity pattern, counseling intervention - case counseling mode as well as the school, family and society [25].But due to China's sexual reproductive health education later than western's, so there may be the impact on the traditional culture and the wholesale westernization, such as stereotypes, separation of teaching and education. Through the research on the college students psychological characteristics, we grasp the psychological development characteristics, to formulate conforms to the university students' sexual health education mode, mainly including classroom, case teaching mode, the combination of daily consultation, crisis intervention, peer education, is now at the research stage. We hope the college students by sexual health education can better adapt a series of mental changes brought by physiological mature, in order to further the good psychological foundation for socialization.

\section{References}

[1]SUI Ping. Survey on gender differences of College students' Sexual psychology and sexual behavior[J] .Chinese Journal of Behavioral Medical Science, 2005,14( 8) : 724.

[2]SONG Shuwen, SUN Ruting et al. Dictionary of psychology[M]: guangxi people press, $1984 ; 174$.

[3]ZHANG Jinfu. Modern youth psychology[M]. Chongqing press.2002;95.

[4]LIU Anping. 278 college students perceptions of teenagers when to sexual health education [J].Chinese Journal of School Doctor. 2004,18(3): 231-232.

[5]ZHANG Peichao, Chi xinli,Wu Mingxia. A Study of Characteristics and Relationship Among Sexual Health Knowledge S Sexual Attitude and Sex-related Behavior in Chinese College Students[J]. Chinese Journal of Clinical Psychology. 2012,20( 6) : 849- 853.

[6]FENG Peng, YAN Lijuan, ZHANG Lu, etc. Investigation and Study on Education Present Situation of College Students in Our School[J].China Higher Medical Education. 2010( 6) : 13 -14 .

[7]HU Yukun. Investment in China's Youth Sexual and Reproductive Health: policy choices of global era[J]. Population and Development. 2010,16( 3) : 27 -28.

[8]The Ministry of Health of The People's Republic of China, The United Nations Programme on HIV, The World Health Organization. Estimation of China's AIDS Epidemic In 2011[J]. Chinese Journal of AIDS \& STD. 2012,18(1) :1 - 5. 
[9]WHO. Addressing the Sexual and Reproductive Health of Adolescents[R].Geneva: WHO,2002: $1-3$.

[10]PITTMAN V, GAHUNGU A. Comprehensive Sexuality Education or Abstinence-only education, Which is More Effective? [J]. J Res Edu-cat Lead,2006,3(2) : 60 -91.

[11]ALFORD S,BRIDGES E,GONZALEZ T, et al. Science and Success: Sex Education and Other Programs that Work to Prevent Teen Pregnancy, HIV \& Sexually transmitted infections[M].2nd ed. Washington,DC: Advocates for youth,2008: 3 -45.

[12]XIAO Bing. To Discuss the Necessity and Mode of College students' sexual health Education[J].Health Vocational Education. 2008(5):28-29

[13]WU Yang. . Research on The Reform and Perfection of The College Students' sexual Health Education[J].The Medical Forum. 2007,11(10):931-933.

[14]WU Yang. A Study on the Pattern and Content of Sexual Health Education for College Student[J]. Zhejiang Journal of Preventive Medicine. 2008,20(4) :3-5.

[15]BAI Jian. The Investigation on Sexual Health Education Question of The Shanxi College Students[J].Chinese Journal of Human Sexuality,2010,19(12) : 27 -28.

[16]LIU Kun, Zhang bing, Tang guizhong. . Investigation and Analysis of Sexual Health Education Present Situation of College Students in Chongqing Area[J].Chongqing Medicine. 2009,38(11) : $1279-1280$.

[17]FANG Gang. Thinking Sexual Education Mode of University-To Discuss Abstinence-only Sex Education and Comprehensive Sex Education[J].China Youth Study. 2008,( 7 ):72-75.

[18]FANG Gang. To Enrich School Sex Education by Using Sex Concept of Human Rights and Social Gender[M] . Taiwan, China: All press, 2011.

[19]PENG Tao. Adolescent Sex Education from The Social Gender Perspective[J]. Chinese Journal of Human Sexuality. 2012,21(8):67-68.

[20]ZHONG Ping. Exploration of Sexual Health Education Pattern in Colleges[J]. Northern Economy and Trade. 2013, (6) :122-123. 